A P PENDIX C

\title{
Wild Leaves Used by Modern Villages
}

TABLE 7 Wild Leaves Used, by Village

\begin{tabular}{|c|c|c|c|c|c|c|c|}
\hline & & $\begin{array}{l}\text { Banda- } \\
\text { Ahenkro }\end{array}$ & Bui & Dorbor & Dumpofie & Makala & Nyiire \\
\hline $\begin{array}{l}\text { Nafaanra/ } \\
\text { Twi name }\end{array}$ & $\begin{array}{l}\text { Scientific } \\
\text { name/Gloss }\end{array}$ & & & $\mathrm{X}$ & & $\mathrm{X}$ & \\
\hline Ayoyo & & & & & & $\mathrm{X}$ & \\
\hline Bobnondele & & $\mathrm{X}$ & & $\mathrm{X}$ & $\mathrm{X}$ & & \\
\hline Bobono & & $\mathrm{X}$ & & & & & \\
\hline Bokoboko & & $\mathrm{X}$ & & & & & \\
\hline Bombo & $\begin{array}{l}\text { Cassia } \\
\text { occidentalis }\end{array}$ & $\mathrm{X}$ & & & & $\mathrm{X}$ & \\
\hline $\begin{array}{l}\text { Bonku/ } \\
\text { Gbonku }\end{array}$ & & $\mathrm{X}$ & & $\mathrm{X}$ & $\mathrm{X}$ & & \\
\hline Chasigbつد & $\begin{array}{l}\text { Ocimum } \\
\text { gratissimum }\end{array}$ & & & $\mathrm{X}$ & $\mathrm{X}$ & & $\mathrm{X}$ \\
\hline Diagyakunu & & $\mathrm{X}$ & & & $\mathrm{X}$ & & \\
\hline Diko & & & & $\mathrm{X}$ & & & \\
\hline Floe/Froe & $\begin{array}{l}\text { Cissus } \\
\text { populnea }\end{array}$ & $\mathrm{X}$ & $\mathrm{X}$ & $\mathrm{X}$ & $\mathrm{X}$ & $\mathrm{X}$ & $\mathrm{X}$ \\
\hline Flewe & $\begin{array}{l}\text { Pavetta } \\
\text { crassipes }\end{array}$ & & & $\mathrm{X}$ & & $\mathrm{X}$ & \\
\hline Flongbe & & $\mathrm{X}$ & & & & & \\
\hline Flonye & $\begin{array}{l}\text { Corchorus } \\
\text { tridens }\end{array}$ & $\mathrm{X}$ & $\mathrm{X}$ & $\mathrm{X}$ & $\mathrm{X}$ & $\mathrm{X}$ & $\mathrm{X}$ \\
\hline FOJli & $\begin{array}{l}\text { Corchorus } \\
\text { olitorius }\end{array}$ & $\mathrm{X}$ & $\mathrm{X}$ & $\mathrm{X}$ & & $\mathrm{X}$ & \\
\hline
\end{tabular}


TABLE 7 (Continued)

\begin{tabular}{|c|c|c|c|c|c|c|c|}
\hline & & $\begin{array}{l}\text { Banda- } \\
\text { Ahenkro }\end{array}$ & Bui & Dorbor & Dumpofie & Makala & Nyiire \\
\hline FumbE & $\begin{array}{l}\text { Vitex } \\
\text { doniana }\end{array}$ & $\mathrm{X}$ & & & & $\mathrm{X}$ & \\
\hline Gabuwere & Wild onion & $\mathrm{X}$ & & & $\mathrm{X}$ & & \\
\hline Gbanja flonye & & $\mathrm{X}$ & & $\mathrm{X}$ & $\mathrm{X}$ & $\mathrm{x}$ & \\
\hline Gboli & & & & $\mathrm{X}$ & & & \\
\hline Gbulo & & $\mathrm{X}$ & & $\mathrm{X}$ & & & $\mathrm{X}$ \\
\hline Hanyini jlon & $\begin{array}{l}\text { Trema } \\
\text { orientalis }\end{array}$ & & & & & & \\
\hline Jamanyiri & Moringa sp. & & & $\mathrm{X}$ & & & \\
\hline Jangboro & & $\mathrm{X}$ & & & & $\mathrm{X}$ & \\
\hline Jelija & $\begin{array}{l}\text { Hibiscus } \\
\text { cannabinus }\end{array}$ & $\mathrm{X}$ & $\mathrm{X}$ & $\mathrm{X}$ & & $\mathrm{X}$ & $\mathrm{X}$ \\
\hline Jyakuno & & & & $\mathrm{X}$ & $\mathrm{X}$ & $\mathrm{x}$ & \\
\hline Jyangburu & & & & $\mathrm{X}$ & $\mathrm{X}$ & & $\mathrm{X}$ \\
\hline $\begin{array}{l}\text { Kafapoe/ } \\
\text { kafapuwee }\end{array}$ & & & & $\mathrm{X}$ & & $\mathrm{X}$ & \\
\hline Kalameshia & & $\mathrm{X}$ & & & $\mathrm{x}$ & & \\
\hline Kambgee & & & & $\mathrm{X}$ & & & \\
\hline Kemeshiama & & $\mathrm{X}$ & & & & & \\
\hline KlaklokagbEE & $\begin{array}{l}\text { Laportea } \\
\text { aestuans }\end{array}$ & & & $\mathrm{X}$ & $\mathrm{X}$ & & \\
\hline $\begin{array}{l}\text { KlandEE } \\
\text { Klododu }\end{array}$ & $\begin{array}{l}\text { Cissus sp. } \\
\text { Uvaria } \\
\text { chamae }\end{array}$ & $\mathrm{X}$ & & & $\mathrm{x}$ & $\mathrm{X}$ & $\mathrm{X}$ \\
\hline Kokoyerere & & & & $\mathrm{X}$ & $\mathrm{x}$ & $\mathrm{x}$ & \\
\hline Koo & & & & & & $\mathrm{x}$ & \\
\hline Kpankpan & $\begin{array}{l}\text { cf. Hymecardia } \\
\text { acida }\end{array}$ & & & & & & \\
\hline Kputukputu & & $\mathrm{X}$ & & & & & \\
\hline Kuka/Gbongbo & $\begin{array}{l}\text { Adansonia } \\
\text { digitata }\end{array}$ & $\mathrm{X}$ & $\mathrm{X}$ & $\mathrm{X}$ & $\mathrm{X}$ & $\mathrm{X}$ & \\
\hline Kusumpura & & & & $\mathrm{X}$ & & & \\
\hline Lakro & $\begin{array}{l}\text { Bridelia } \\
\text { feruginea }\end{array}$ & & & & & & \\
\hline$L O m$ & & $\mathrm{X}$ & $\mathrm{X}$ & $\mathrm{X}$ & $\mathrm{X}$ & $\mathrm{X}$ & \\
\hline \multicolumn{2}{|c|}{ Lombaoe/Longbowere } & $\mathrm{X}$ & & $\mathrm{X}$ & & & \\
\hline Mbandele & & & & & & & $\mathrm{X}$ \\
\hline Moliere & & & & & $\mathrm{X}$ & & \\
\hline
\end{tabular}


TABLE 7 (Continued)

\begin{tabular}{|c|c|c|c|c|c|c|c|}
\hline & & $\begin{array}{l}\text { Banda- } \\
\text { Ahenkro }\end{array}$ & Bui & Dorbor & Dumpofie & Makala & Nyiire \\
\hline \multirow[t]{2}{*}{ Nafaawere } & & $\mathrm{X}$ & & & & & \\
\hline & $\begin{array}{l}\text { Ocimum } \\
\text { basilicum }\end{array}$ & X & & $\mathrm{X}$ & $\mathrm{X}$ & $\mathrm{X}$ & $\mathrm{X}$ \\
\hline Ndele & & $\mathrm{X}$ & & & & & \\
\hline Ngasingasi & & & & & $\mathrm{X}$ & & \\
\hline Ngbrengbre & & & & $\mathrm{X}$ & & & \\
\hline Ngoli & & & & $\mathrm{X}$ & $\mathrm{X}$ & $\mathrm{X}$ & \\
\hline \multicolumn{7}{|l|}{ Ngunocho- } & \\
\hline Nom & & & $\mathrm{X}$ & & & & \\
\hline Nyanya & & $\mathrm{X}$ & & & & & $\mathrm{X}$ \\
\hline NyiEE & & $\mathrm{X}$ & $\mathrm{X}$ & $\mathrm{X}$ & & $\mathrm{X}$ & $\mathrm{X}$ \\
\hline Mbabluwi & & $\mathrm{X}$ & & & & & \\
\hline Peelolo & $\begin{array}{l}\text { Parinari } \\
\text { curatellifolia }\end{array}$ & & & & & & \\
\hline Shia & $\begin{array}{l}\text { Ficus } \\
\text { gnaphalocarpa }\end{array}$ & & & & & $\mathrm{X}$ & \\
\hline Sugbosemna & & & & & & $\mathrm{X}$ & \\
\hline Sulom & & & & $\mathrm{X}$ & & & \\
\hline Takatumo & & $\mathrm{X}$ & & & & & \\
\hline Yomaa & $\begin{array}{l}\text { Piliostigma } \\
\text { thonningii }\end{array}$ & & & & & & \\
\hline Zinblekole & & $\mathrm{X}$ & & & & & \\
\hline Zizibi & & & & $\mathrm{X}$ & & & \\
\hline Total leaves $n$ & eed, by village & 32 & 8 & 31 & 20 & 23 & 11 \\
\hline
\end{tabular}

